\title{
Aggregate modeling and analysis of air conditioning load using coupled Fokker-Planck equations
}

\author{
Dingyi CHENG ${ }^{1}$, Wen ZHANG $^{1}{ }^{\mathbb{D}}$, Yutian LIU $^{1}$
}

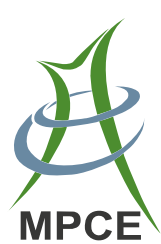

\begin{abstract}
Demand response has gained significant attention recently with the increasing penetration of renewable energy sources in power systems. Air conditioning loads are typical thermostatically controlled loads which can play an active role in ancillary services by regulating their aggregated power consumption. The aggregation of air conditioners is essential to the control of air conditioning loads. In this paper, linear state equations are proposed to aggregate air conditioning loads by solving coupled Fokker-Planck equations (CFPEs) using the finite difference method. By analyzing the numerical stability and convergence of the difference scheme, the grid spacings, including temperature step and time step, are properly determined according to the maximal principle. Stationary solutions of the CFPEs are obtained by analytical and numerical methods. Furthermore, a classification method using dimension reduction is proposed to deal with the problem of heterogeneous parameters and interval estimation is
\end{abstract}

CrossCheck date: 7 December 2017

Electronic supplementary material The online version of this article (https://doi.org/10.1007/s40565-018-0396-2) contains supplementary material, which is available to authorized users.

Received: 10 December 2016/Accepted: 7 December 2017/Published online: 20 March 2018

(C) The Author(s) 2018

Wen ZHANG

zhangwen@sdu.edu.cn

Dingyi CHENG

dingyi_cheng@mail.sdu.edu.cn

Yutian LIU

liuyt@sdu.edu.cn

1 Key Laboratory of Power System Intelligent Dispatch and Control of Ministry of Education, Shandong University, Jinan 250061, China applied to describe the stochastic behavior of air conditioning loads. The simulation results verify the effectiveness of the proposed methods.

Keywords Aggregate model, Air conditioner, Coupled Fokker-Planck equation, Grid spacing selection, Heterogeneous, Interval estimation, Linear state equation, Stationary solution

\section{Introduction}

Maintaining the balance between the power generation and the demand is a key issue in power system operation. Traditionally, the generation side and interconnection lines are primarily used for this purpose [1]. With the increased penetration of renewable energy sources, conventional control strategies may be not enough to respond to the uncertainty of these renewable energy sources. Demand response has potential to help match the supply and the demand of power systems owing to the flexibility of controllable loads [2, 3]. Because thermostatically controlled loads (TCLs) can store energy in the form of temperature gradients, they represent a promising end-use category to engage in power system services [4]. Air conditioners are typical TCLs. The percentage of energy consumed by air conditioners of buildings in a city, like Jeddah, Saudi Arabia, is even over $50 \%$ of the total electric energy during the summer [5]. Applying appropriate control to air conditioners will play an important role in energy conservation and power system control [6-9].

Aggregation of air conditioners is essential to the control of air conditioners. To simulate the behavior of the air conditioner population, the most direct method is to aggregate thousands of air conditioners. In [10], the Monte 
Carlo method combined with smoothing techniques was proposed for numerical simulations. However, this would be computationally intensive and the aggregate system would not be in a form amenable to many control techniques. To facilitate the control of air conditioners, the main idea is to establish the aggregate model, which can characterize the temperature probability density evolution of the air conditioner population. In [11] and [12], the statebin-based 1st-order TCL model was proposed and the state space matrix was identified by a Kalman filter. However, this identification approach may not converge when the number of states becomes large and it may not perform well for online estimation [13]. In [7] and [13], state-binbased 1st-order TCL models considering the device lockout time were proposed for different control purposes. The state-bin-based heterogeneous 2nd-order TCL model was proposed in [14]. Besides, some aggregate models that did not consider the temperature probability density evolution have been proposed and received extensive concern. The aggregate duty cycle was used to aggregate TCLs in $[15,16]$. This method was often applied to primary frequency control. In [17], the proposed model analytically characterized the aggregate power response of the step change in temperature set points. In [8], a population of TCLs was modeled as a battery. In [18], a virtual power plant model was applied to aggregate TCLs.

However, all of these models neglected the randomness of air conditioning loads. Coupled Fokker-Planck equations (CFPEs) were applied to aggregation of TCLs considering the randomness in [19]. It should be noted that the above methods mainly focused on point estimation which is inadequate to describe the stochastic behavior in aggregating air conditioning loads.

The stationary solutions of CFPEs are important because they can provide the initial and final conditions for state equations. In [20], Laplace transformation and inverse transformation were applied to the simplified CFPEs and the analytical stationary solutions were given. Reference [21] obtained the form of analytical stationary solutions of CFPEs by analyzing their eigenvalues and eigenfunctions. However, the coefficients of the analytical solutions were not provided in analytical form. Therefore, they can only be solved by numerical methods. The stationary solutions need to be developed further to be useful for real-time control.

Time-domain solutions of CFPEs are essential to the aggregation of air conditioning loads. CFPEs are linear second-order parabolic partial differential equations with coupled boundary conditions. As the analytical solutions cannot be obtained in time domain at present, numerical solutions are necessary. The finite difference method (FDM) is effective in solving partial differential equations, and correct grid spacings, including temperature step and time step, are essential because the improper grid spacings will lead to inaccurate solutions. In [22], the grid spacing was selected for the linear second-order parabolic partial differential equation with defined boundary conditions. The boundary conditions of CFPEs for aggregating air conditioning loads are coupled, which means the results reported in [22] cannot be used directly. The grid spacing selection of CFPEs should receive careful attention.

The main contributions of this paper are as follows:

1) The linear state equations, which characterize the temperature probability density evolution of the air conditioner population considering randomness, are proposed by solving CFPEs using the FDM to aggregate air conditioning loads.

2) By analyzing the numerical stability and convergence of the difference scheme of CFPEs, the grid spacings are properly determined.

3) Both analytical and numerical methods are proposed to get stationary solutions of CFPEs.

4) A classification method using dimension reduction is proposed for heterogeneous loads.

5) Interval estimation is introduced to reflect the stochastic behavior of air conditioning loads.

The rest of the paper is organized as follows. In Section 2, a stochastic space thermal model for air conditioners is described. The aggregate model using FokkerPlanck equations is presented in Section 3. The aggregate model for heterogeneous loads is presented in Section 4. Simulation results are shown in Section 5 to verify the methods proposed in previous sections. Finally, conclusions are drawn in Section 6.

\section{Stochastic individual load model}

The dynamic model for temperature of a room with air conditioners can be expressed by a first order differential equation which describes the relationship between the indoor temperature, the outdoor temperature and the rate of energy transfer from outdoors to indoors by the air conditioner. Compared with higher order models, it is relatively easy to apply control strategies to an air-conditioning system by using a first order model with fewer parameters. The first order model shown in (1) describes the thermal process [23].

$\frac{\mathrm{d} x}{\mathrm{~d} t}=\frac{K}{C}\left(x_{o}-x\right)+\frac{s \alpha P_{e}}{C}$

where $x$ is the temperature inside the space $\left({ }^{\circ} \mathrm{C}\right) ; x_{o}$ is the temperature outside the space $\left({ }^{\circ} \mathrm{C}\right) ; C$ is the thermal capacity of the space $\left(\mathrm{J} /{ }^{\circ} \mathrm{C}\right) ; K$ is the thermal conductance of the space $\left(\mathrm{W} /{ }^{\circ} \mathrm{C}\right) ; P_{e}$ is the electrical power generated by 
air conditioners inside the space (W); $s$ is the value stands for working mode of air conditioners, for heating mode $s=1$ and for cooling mode $s=-1 ; \alpha$ is the coefficient of performance (COP) of air conditioners inside the space and the thermal power generated by the air condition inside the space $P_{h}=s \alpha P_{e} ; t$ is the time (second).

The electrical power used by air conditioners inside the space is related to the thermostat state $m$, a dimensionless discrete variable equal to 0 (off) or 1 (on). That is:

$P_{e}=m P_{N}$

where $P_{N}$ is the rated power of the air conditioner.

The evolution of the discrete state $m$ is governed by the thermostat with temperature setting $x_{\text {set }}$ and dead band $\delta$. That is:

$$
m\left(t_{n+1}\right)= \begin{cases}1 & s\left[x-\left(x_{\text {set }}-\frac{s \delta}{2}\right)\right]<0 \\ 0 & s\left[x-\left(x_{\text {set }}+\frac{s \delta}{2}\right)\right]>0 \\ m\left(t_{n}\right) & \text { otherwise }\end{cases}
$$

To facilitate the aggregation of air conditioners, stochastic heat gain or heat loss (such as fluctuating number of people in the residence, doors and windows being opened and closed, and appliances being used) should be considered on the basis of the space thermal model [24]. A stochastic space thermal model, which is a stochastic differential equation can be formulated as:

$\mathrm{d} x=\left[\frac{K}{C}\left(x_{o}-x\right)+\frac{s \alpha P_{e}}{C}\right] \mathrm{d} t+\mathrm{d} v_{t}$

where $v_{t}$ is a Wiener process with variance parameter $\sigma^{2}$.

\section{Aggregate model of homogeneous loads using Fokker-Planck equations}

\subsection{Description of Fokker-Planck equations}

By considering the probability distribution (by temperature) of a population of air conditioners and making the assumption that the population is homogeneous, Malhamé and Chong (M\&C) [19] proved that it is possible to construct a system of CFPEs to describe the dynamics of the population. This paper takes the cooling mode as the working mode of air conditioners, and the main equations of M\&C's model can be written in continuous time as follows:

$$
\begin{aligned}
& \frac{\partial f_{1}(x, t)}{\partial t}=\frac{\partial F_{1}(x) f_{1}(x, t)}{\partial x}+\frac{\sigma^{2}}{2} \frac{\partial^{2} f_{1}(x, t)}{\partial x^{2}} \\
& \frac{\partial f_{0}(x, t)}{\partial t}=\frac{\partial F_{0}(x) f_{0}(x, t)}{\partial x}+\frac{\sigma^{2}}{2} \frac{\partial^{2} f_{0}(x, t)}{\partial x^{2}}
\end{aligned}
$$

where $f_{1}(x, t)$ and $f_{0}(x, t)$ are the probability densities of loads in the on and off states, respectively; $F_{1}(x)=\left[K\left(x-x_{0}\right)+\alpha P_{N}\right] / C \quad$ and $\quad F_{0}(x)=K\left(x-x_{0}\right) /$ $C$ are the negative gradient of temperature inside the space in the on and off states, respectively.

The temperature range $(-\infty,+\infty)$ is divided into three temperature regions: region $a\left(-\infty, x_{-}\right]$, region $b\left[x_{-}, x_{+}\right]$and region $c\left[x_{+},+\infty\right)$, where $x_{-}=x_{\text {set }}-\delta / 2$ is the temperature lower limit and $x_{+}=x_{\text {set }}+\delta / 2$ is the temperature upper limit. The following boundary conditions must hold for all $t$.

Absorbing boundaries:

$f_{1 b}\left(x_{-}, t\right)=f_{0 b}\left(x_{+}, t\right)=0$

where $f_{0 b}$ and $f_{1 b}$ are the probability densities of loads of region $b$ in the off and on states, respectively.

Conditions at infinity:

$f_{0 a}(-\infty, t)=f_{1 c}(+\infty, t)=0$

where $f_{0 a}$ is the probability density of loads of region $a$ in the off state; $f_{1 c}$ is the probability density of loads of region $c$ in the on state.

Continuity conditions:

$f_{0 a}\left(x_{-}, t\right)=f_{0 b}\left(x_{-}, t\right)$

$f_{1 b}\left(x_{+}, t\right)=f_{1 c}\left(x_{+}, t\right)$

Probability conservation:

$-\frac{\partial f_{0 a}\left(x_{-}, t\right)}{\partial x}+\frac{\partial f_{0 b}\left(x_{-}, t\right)}{\partial x}+\frac{\partial f_{1 b}\left(x_{-}, t\right)}{\partial x}=0$

$\frac{\partial f_{1 c}\left(x_{+}, t\right)}{\partial x}-\frac{\partial f_{1 b}\left(x_{+}, t\right)}{\partial x}-\frac{\partial f_{0 b}\left(x_{+}, t\right)}{\partial x}=0$

Because of the probability conservation conditions, the integrated probability density will always be unity, that is:

$$
\begin{aligned}
& \int_{-\infty}^{x_{-}} f_{0 a}(x, t) \mathrm{d} x+\int_{x_{-}}^{x_{+}}\left(f_{0 b}(x, t)+f_{1 b}(x, t)\right) \mathrm{d} x \\
& \quad+\int_{x_{-}}^{x_{+}} f_{1 c}(x, t) \mathrm{d} x=\int_{-\infty}^{x_{-}} f_{0 a}(x, 0) \mathrm{d} x \\
& \quad+\int_{x_{-}}^{x_{+}}\left(f_{0 b}(x, 0)+f_{1 b}(x, 0)\right) \mathrm{d} x+\int_{x_{-}}^{x_{+}} f_{1 c}(x, 0) \mathrm{d} x=1
\end{aligned}
$$

The total number of air conditioners is $N_{a c}$. At time $t$, the average proportion of air conditioners in the on state is:

$\bar{m}(t)=\int_{x_{-}}^{x_{+}} f_{1 b}(x, t) \mathrm{d} x+\int_{x_{-}}^{x_{+}} f_{1 c}(x, t) \mathrm{d} x$

The operating characteristics of air conditioners are independent of one another. So the number of air conditioners in the on state $X(t)$ is subject to the binomial distribution $X(t) \sim B\left(N_{a c}, \bar{m}(t)\right)$. The expected value and variance of $X(t)$ are: 
$E(X(t))=N_{a c} \bar{m}(t)$

$D(X(t))=N_{a c} \bar{m}(t)(1-\bar{m}(t))$

\subsubsection{Point estimation}

The average aggregate power of air conditioners is:

$\bar{P}(t)=E(X(t)) P_{N}$

This provides a point estimate for aggregation.

\subsubsection{Interval estimation}

The aggregate power of air conditioners $P(t)$ is a random variable. Interval estimation is needed, which is given by:

$P_{r}\left(P_{1}(t) \leq P(t) \leq P_{2}(t)\right)=\gamma$

where $P_{1}(t)$ and $P_{2}(t)$ denote the lower and upper confidence limits of aggregate power, respectively; $\gamma \in(0,1)$ is the confidence level; $P_{r}(\cdot)$ is the probability function. It is not convenient to use the binomial distribution directly in interval estimation, because the calculation of probability is complicated especially when $N_{a c}$ is large. The normal distribution is suitable for interval estimation and $X(t)$ has approximately a normal distribution $N(E(X(t)), D(X(t)))$ because the binomial distribution can be approximated by the normal distribution [25]. In practice, the approximation is adequate provided that both $N_{a c} \bar{m}(t) \geq 10$ and $N_{a c}(1-\bar{m}(t)) \geq 10$, and then interval estimates can be obtained easily. Two common used interval estimates are given below:

$$
\begin{aligned}
& P_{r}(E(X(t))-2 \sqrt{D(X(t))} \leq X(t) \\
& \quad \leq E(X(t))+2 \sqrt{D(X(t))}) \approx 95.4 \% \\
& P_{r}(E(X(t))-3 \sqrt{D(X(t))} \leq X(t) \\
& \quad \leq E(X(t))+3 \sqrt{D(X(t))}) \approx 99.7 \%
\end{aligned}
$$

The interval estimate for homogeneous loads is (18).

The average proportion $\bar{m}(t)$ is important for either point estimation or interval estimation. However, exact solutions for the CFPEs are difficult to obtain. Appropriate numerical methods should be used to solve the CFPEs.

\subsection{Time-domain solutions of CFPEs using FDM}

CFPEs are partial differential equations, so the FDM can be applied to solve them. The temperature range of $(-\infty$, $+\infty)$ is impossible for numerical calculation, so the temperature range can be set as $\left[x_{\min }, x_{\max }\right]$, where probability densities of loads in $x<x_{\min }$ and $x>x_{\max }$ are 0 consistently with their limiting trend. The time step is $\tau=t_{j+1}-t_{j}$ and the temperature step is $h=x_{i+1}-x_{i}$. There are $N_{a}+1, N_{b}+1$ and $N_{c}+1$ temperature points in region $a\left[x_{\min }, x_{-}\right]$, region $b\left[x_{-}, x_{+}\right]$and region $c\left[x_{+}\right.$, $\left.x_{\max }\right]$, respectively, and there are $N+1$ time points. The implicit central finite-difference scheme [26] is applied to solve CFPEs. At interior temperature points,

$$
\begin{aligned}
\frac{f_{i, j+1}-f_{i, j}}{\tau}= & \frac{1}{2}\left(\frac{K}{C} f_{i, j}+F\left(x_{i}\right) \frac{f_{i+1, j}-f_{i-1, j}}{2 h}\right. \\
& \left.+\frac{\sigma^{2}}{2} \frac{f_{i+1, j}+f_{i-1, j}-2 f_{i, j}}{h^{2}}\right)+\frac{1}{2}\left(\frac{K}{C} f_{i, j+1}+F\left(x_{i}\right)\right. \\
& \left.\frac{f_{i+1, j+1}-f_{i-1, j+1}}{2 h}+\frac{\sigma^{2}}{2} \frac{f_{i+1, j+1}+f_{i-1, j+1}-2 f_{i, j+1}}{h^{2}}\right)
\end{aligned}
$$

where $f_{i, j}=f_{m}\left(x_{i}, t_{j}\right)$ is the probability density of loads in state $m$, and state $m$ does not appear in these equations because it does not affect their form; $F\left(x_{i}\right)=F_{m}\left(x_{i}\right)$ is the negative temperature gradient inside the space in the $m$ state.

At boundary temperature points, according to finite volume method [26],

$$
\begin{aligned}
\frac{f_{0, j+1}-f_{0, j}}{\tau}= & \frac{1}{2}\left[\frac{K}{C} f_{0, j}+F\left(x_{0}\right) \frac{f_{1, j}-f_{0, j}}{h}\right. \\
& \left.+\frac{\sigma^{2}}{h}\left(\frac{f_{1, j}-f_{0, j}}{h}-\frac{\partial f_{0, j}}{\partial x}\right)\right]+\frac{1}{2}\left[\frac{K}{C} f_{0, j+1}+F\left(x_{0}\right)\right. \\
& \left.. \frac{f_{1, j+1}-f_{0, j+1}}{h}+\frac{\sigma^{2}}{h}\left(\frac{f_{1, j+1}-f_{0, j+1}}{h}-\frac{\partial f_{0, j+1}}{\partial x}\right)\right] \\
\frac{f_{N, j+1}-f_{N, j}}{\tau}= & \frac{1}{2}\left[\frac{K}{C} f_{N, j}+F\left(x_{N}\right) \frac{f_{N, j}-f_{N-1, j}}{h}\right. \\
& \left.+\frac{\sigma^{2}}{h}\left(-\frac{f_{N, j}-f_{N-1, j}}{h}+\frac{\partial f_{0, j}}{\partial x}\right)\right]+\frac{1}{2}\left[\frac{K}{C} f_{N, j+1}+F\left(x_{N}\right)\right. \\
& \left.. \frac{f_{N, j+1}-f_{N-1, j+1}}{h}+\frac{\sigma^{2}}{h}\left(-\frac{f_{N, j+1}-f_{N-1, j+1}}{h}+\frac{\partial f_{N, j+1}}{\partial x}\right)\right]
\end{aligned}
$$

$f_{i, j}, \partial f_{0, j+1} / \partial x$ and $\partial f_{N, j+1} / \partial x$ are the unknown variables of which the total number is $N_{a}+2 N_{b}+N_{c}+12$.

Absorbing boundary conditions:

$f_{1 b}\left(x_{0}, t_{j}\right)=f_{0 b}\left(x_{N_{b}}, t_{j}\right)=0$

where $x_{0}$ is the initial point of each region and $x_{0}$ is not same in each region.

Conditions at infinity:

$2 F_{0}\left(x_{0}\right) f_{0 a}\left(x_{0}, t_{j}\right)+\sigma^{2} \frac{\partial f_{0 a}\left(x_{0}, t_{j}\right)}{\partial x}=0$
$2 F_{1}\left(x_{N_{c}}\right) f_{1 c}\left(x_{N_{c}}, t_{j}\right)+\sigma^{2} \frac{\partial f_{1 c}\left(x_{N_{c}}, t_{j}\right)}{\partial x}=0$

Continuity conditions: 
$f_{0 a}\left(x_{N_{a}}, t_{j}\right)=f_{0 b}\left(x_{0}, t_{j}\right)$

$f_{1 b}\left(x_{N_{b}}, t_{j}\right)=f_{1 c}\left(x_{0}, t_{j}\right)$

Probability conservation:

$-\frac{\partial f_{0 a}\left(x_{N_{a}}, t_{j}\right)}{\partial x}+\frac{\partial f_{0 b}\left(x_{0}, t_{j}\right)}{\partial x}+\frac{\partial f_{1 b}\left(x_{0}, t_{j}\right)}{\partial x}=0$

$\frac{\partial f_{1 c}\left(x_{0}, t_{j}\right)}{\partial x}-\frac{\partial f_{1 b}\left(x_{N_{b}}, t_{j}\right)}{\partial x}-\frac{\partial f_{0 b}\left(x_{N_{b}}, t_{j}\right)}{\partial x}=0$

By linear transformation, 8 partial differential variables, 2 variables at absorbing boundaries and 2 of the variables in continuity conditions can be eliminated.

At every time step, there are $N_{a}+2 N_{b}+N_{c}$ equations and every equation is linear equation. The following linear equation system can be obtained:

$\boldsymbol{A}_{1} \boldsymbol{f}\left(t_{j+1}\right)=\boldsymbol{A}_{2} \boldsymbol{f}\left(t_{j}\right)$

$\boldsymbol{f}\left(t_{j}\right)=\left[\begin{array}{llll}\boldsymbol{f}_{0 a}^{\mathrm{T}}\left(t_{j}\right) & \boldsymbol{f}_{0 b}^{\mathrm{T}}\left(t_{j}\right) & \boldsymbol{f}_{1 b}^{\mathrm{T}}\left(t_{j}\right) & \boldsymbol{f}_{1 c}^{\mathrm{T}}\left(t_{j}\right)\end{array}\right]^{\mathrm{T}}$

$\boldsymbol{f}_{0 a}\left(t_{j}\right)=\left[\begin{array}{lll}f_{0 a}\left(x_{0}, t_{j}\right) & \cdots & f_{0 a}\left(x_{N_{a}}, t_{j}\right)\end{array}\right]^{\mathrm{T}}$

$\boldsymbol{f}_{0 b}\left(t_{j}\right)=\left[\begin{array}{lll}f_{0 b}\left(x_{1}, t_{j}\right) & \cdots & f_{0 b}\left(x_{N_{b}-1}, t_{j}\right)\end{array}\right]^{\mathrm{T}}$

$\boldsymbol{f}_{1 b}\left(t_{j}\right)=\left[\begin{array}{lll}f_{1 b}\left(x_{1}, t_{j}\right) & \cdots & f_{1 \mathrm{~b}}\left(x_{N_{b}-1}, t_{j}\right)\end{array}\right]^{\mathrm{T}}$

$\boldsymbol{f}_{1 c}\left(t_{j}\right)=\left[\begin{array}{lll}f_{1 c}\left(x_{0}, t_{j}\right) & \cdots & f_{1 \mathrm{c}}\left(x_{N_{c}}, t_{j}\right)\end{array}\right]^{\mathrm{T}}$

The derivation of $\boldsymbol{A}_{1}$ and $\boldsymbol{A}_{2}$ is shown in the Appendix A. The number of unknown variables is also $N_{a}+2 N_{b}+N_{c}$, and accordingly, unique solutions can be obtained.

The finite volume method [26] is used to implement the difference schemes for boundary temperature points, and the total probability in the system will always be unity due to the trapezoidal rule. That is:

$I_{0}\left(t_{j}\right)=\frac{h}{2}\left(f_{0 a}\left(x_{0}, t_{j}\right)+2 \sum_{i=1}^{N_{a}} f_{0 a}\left(x_{i}, t_{j}\right)+2 \sum_{i=1}^{N_{b}-1} f_{0 b}\left(x_{i}, t_{j}\right)\right)$

$I_{1}\left(t_{j}\right)=\frac{h}{2}\left(2 \sum_{i=1}^{N_{b}-1} f_{1 b}\left(x_{i}, t_{j}\right)+2 \sum_{i=0}^{N_{c}-1} f_{1 c}\left(x_{i}, t_{j}\right)+f_{1 c}\left(x_{N_{c}}, t_{j}\right)\right)$

$I_{0}\left(t_{j+1}\right)+I_{1}\left(t_{j+1}\right)=I_{0}\left(t_{j}\right)+I_{1}\left(t_{j}\right)$

It should be noted that (39) can be derived by the elementary line transformation of (31). And (39) cannot be got by line operation with the loss of any equations of (31).

Furthermore, the linear state equations can be established, which are:
$\boldsymbol{f}\left(t_{j+1}\right)=\boldsymbol{A} \boldsymbol{f}\left(t_{j}\right)$

$\bar{m}\left(t_{j+1}\right)=I_{1}\left(t_{j+1}\right)$

where $\boldsymbol{A}=\boldsymbol{A}_{1}^{-1} \boldsymbol{A}_{2}$.

The aggregate power of air conditioners is:

$\bar{P}\left(t_{j+1}\right)=\bar{m}\left(t_{j+1}\right) N_{a c} P_{N}=I_{1}\left(t_{j+1}\right) N_{a c} P_{N}$

The aggregate modeling of air conditioning loads is shown in (40)-(42), in which linear state equations are developed by solving CFPEs using the FDM.

The aggregate model proposed in this paper can also be applied to many proposed control strategies based on temperature density evolution models. The state space matrixes of many temperature density evolution models were decided by identification of air conditioning loads in the aggregator using a Kalman filter. The state space matrix of proposed aggregate model can be decided using the parameters identified in each room, which is more convenient in on-line application. Some suitable parameter identification methods are available, such as the windowvarying particle filter proposed in [27].

The choice of grid spacings is essential to ensuring the accuracy of solutions. If the grid spacings are too large, the solutions are not accurate, but if too small, the computation time will increase. The stability and convergence criterion of the FDM determine the best grid spacings. We obtain the following criteria according to the maximal principle and they are a sufficient condition for convergence to an accurate solution.

$$
\left\{\begin{array}{l}
2\left(1-\frac{1}{2} \frac{K}{C} \tau\right)-\frac{1}{2} \frac{\tau}{h^{2}}\left(\sigma^{2}-\left|F\left(x_{i}\right)\right|_{\max } h\right)>0 \\
\sigma^{2}-\left|F\left(x_{i}\right)\right|_{\max } h \geq 0 \\
1+\frac{1}{2} \frac{K}{C} \tau-\frac{1}{2} \sigma^{2} \frac{\tau}{h^{2}} \geq 0
\end{array}\right.
$$

where $\left|F\left(x_{i}\right)\right|_{\max }$ is the maximum value of $\left|F_{0}(x)\right|$ for $x \in$ $\left[x_{\min }, x_{+}\right]$and $\left|F_{1}(x)\right|$ for $x \in\left[x_{-}, x_{\max }\right]$.

Equation (43) is the suggested guide to choose grid spacings.

\subsection{Stationary solutions}

It is important to get the stationary solutions of CFPEs, which provide the initial and final conditions for state equations.

\subsubsection{Analytical stationary solutions}

In [21], the author derives the following stationary solutions with 8 coefficients to solve: 


$$
\begin{aligned}
f_{1}(x)= & \mathrm{e}^{-\left(\frac{x-x_{o}+P_{N} R}{\sigma \sqrt{C R}}\right)^{2}} \\
& \cdot\left(a_{01}-\frac{1}{2} a_{11} \operatorname{ierf}\left(\mathrm{i} \frac{x-x_{o}+P_{N} R}{\sigma \sqrt{C R}}\right) \sqrt{\pi C R} \sigma\right) \\
f_{0}(x)= & \mathrm{e}^{-\left(\frac{x-x_{o}}{\sigma \sqrt{C R}}\right)^{2}} \\
& \cdot\left(a_{00}-\frac{1}{2} a_{10} \operatorname{ierf}\left(\mathrm{i} \frac{x-x_{o}}{\sigma \sqrt{C R}}\right) \sqrt{\pi C R} \sigma\right)
\end{aligned}
$$

where $\operatorname{ierf}(\mathrm{i} x)=\frac{2}{\sqrt{\pi}} \int_{0}^{x} \mathrm{e}^{t^{2}} \mathrm{~d} t ; R=1 / K$.

The 8 coefficients are $a_{00 a}, a_{10 a}, a_{00 b}, a_{10 b}, a_{01 b}, a_{11 b}$, $a_{01 c}$ and $a_{11 c}$ which must be separately determined for each temperature region and operating state.

The author uses 9 equations to solve these 8 coefficients, which is feasible in numerical calculation. Two of these 9 equations, which are the conditions at infinity, are satisfied automatically by (44) and (45) and may therefore be ignored. Based on (44) and (45), we provide the completely analytical stationary solutions for CFPEs, which are:

$$
\begin{aligned}
& f_{0 a}(x, \infty) \\
& \quad=a_{10 b} \sqrt{C R} \sigma \int_{\frac{x_{-}-x_{0}}{\sigma \sqrt{C R}}}^{\frac{x_{+}-x_{0}}{\sigma \sqrt{C R}}} \mathrm{e}^{t^{2}-\left(\frac{x-x_{0}}{\sigma \sqrt{C R}}\right)^{2}} \mathrm{~d} t \quad x \in\left(-\infty, x_{-}\right]
\end{aligned}
$$

$$
\begin{aligned}
& f_{0 b}(x, \infty) \\
& =a_{10 b} \sqrt{C R} \sigma \int_{\frac{x-x_{o}}{\sigma \sqrt{C R}}}^{\frac{x_{+}-x_{o}}{\sigma \sqrt{C R}}} \mathrm{e}^{t^{2}-\left(\frac{x-x_{o}}{\sigma \sqrt{C R}}\right)^{2}} \mathrm{~d} t \quad x \in\left[x_{-}, x_{+}\right] \\
& f_{1 b}(x, \infty) \\
& =a_{10 b} \sqrt{C R} \sigma \int_{\frac{x_{-}-x_{o}+P_{N} R}{\sigma \sqrt{C R}}}^{\frac{x-x_{o}+P_{N} R}{\sigma \sqrt{C R}}} \mathrm{e}^{t^{2}-\left(\frac{x-x_{o}+P_{N} R}{\sigma \sqrt{C R}}\right)^{2}} \mathrm{~d} t \quad x \in\left[x_{-}, x_{+}\right]
\end{aligned}
$$

$$
\begin{aligned}
& f_{1 \mathrm{c}}(x, \infty) \\
& \quad=a_{10 b} \sqrt{C R} \sigma \int_{\frac{x_{-}-x_{o}+P_{N} R}{\sigma \sqrt{C R}}}^{\frac{x_{+}-x_{o}+P_{N} R}{\sigma \sqrt{C R}}} \mathrm{e}^{t^{2}-\left(\frac{x-x_{O}+P_{N} R}{\sigma \sqrt{C R}}\right)^{2}} \mathrm{~d} t \quad x \in\left[x_{+},+\infty\right)
\end{aligned}
$$

It should be noted that:

$$
\begin{aligned}
& \int_{-\infty}^{x_{-}} f_{0 a}(x, \infty) \mathrm{d} x+\int_{x_{-}}^{x_{+}}\left(f_{0 b}(x, \infty)+f_{1 b}(x, \infty)\right) \mathrm{d} x \\
& +\int_{x_{-}}^{x_{+}} f_{1 c}(x, \infty) \mathrm{d} x=1
\end{aligned}
$$

The unique $a_{10 b}$ can be decided by substituting (46)(49) to (50) and the stationary solutions can be obtained.
The analytical stationary solutions for CFPEs are (46)(50).

\subsubsection{Numerical stationary solutions}

Analytical solutions are exact but complex integrals need to be calculated. Numerical solutions are required for fast calculation.

From (31), the stationary solutions should satisfy

$\boldsymbol{A}_{1} f(\infty)=\boldsymbol{A}_{2} f(\infty)$

where $f(\infty)$ is the numerical stationary solution. According to the line transformation from (31) to (39), both of the lefthand side (LHS) and right-hand side (RHS) of (51) can be transformed to $I_{0}(\infty)+I_{1}(\infty)$, which also means zero vector can be obtained by line operation of $\boldsymbol{A}_{1}-\boldsymbol{A}_{2}$. In other words, the row vectors of $\boldsymbol{A}_{1}-\boldsymbol{A}_{2}$ are linearly dependent. Zero vector cannot be got by line operation of $\boldsymbol{A}_{1}-\boldsymbol{A}_{2}$ with the loss of any row. Thus the rank of $\boldsymbol{A}_{1}-\boldsymbol{A}_{2}$ is $N_{a}+2 N_{b}+N_{c}-1$ and an additional equation should be added to solve $f(\infty)$. This equation is provided by:

$I_{0}(\infty)+I_{1}(\infty)=1$

By using (52) and the $N_{a}+2 N_{b}+N_{c}-1$ equations of (51), the numerical stationary solutions can be obtained.

\section{Aggregate model of heterogeneous loads}

\subsection{Parameter classification}

This paper considers a realistic scenario in which the parameters of air conditioners are heterogeneous. It will focus on the effect of varying $C, K$ and $P_{N}$, the fundamental parameters of the space thermal model, which are assumed to follow log-normal distributions as in [21]. To deal with load heterogeneity, parameters are classified into several clusters and a homogeneous aggregate model is computed for each cluster. There will be many clusters when these three parameters are classified directly according to varying $C, K$ and $P_{N}$. Dimension reduction can be achieved by defining $p_{1}=K / C$ and $p_{2}=P_{N} / C$ to determine the space thermal model, so that the number of clusters is reduced a lot when classified according to $p_{1}$ and $p_{2}$.

With dimension reduction, two steps are needed when grouping heterogeneous parameters considering reasonable parameter combinations, which means the indoor temperature can approach to the lower limit $x_{-}$in cooling mode (or the upper limit $x_{+}$in heating mode) and the following condition should be satisfied: 
$p_{1}\left[-s x_{\mathrm{o}}+\frac{1}{2}(s-1) x_{-}+\frac{1}{2}(s+1) x_{+}\right] \leq \alpha p_{2}$

Firstly, $n_{1}$ clusters of $p_{1}$ and $p_{2}$ are classified according to the probability density function of $p_{1}$ and $p_{2}$, with weight $w_{i}$ of each cluster as defined by (54)-(56).

$$
\begin{aligned}
w_{i} & \propto f\left(\lg p_{1}, \lg p_{2} \mid p_{1}\left[-s x_{0}+\frac{(s-1) x_{-}}{2}+\frac{(s+1) x_{+}}{2}\right] \leq \alpha p_{2}\right) \\
& \propto f\left(\lg p_{1}, \lg p_{2}\right) \propto \int f\left(\lg p_{1}, \lg p_{2}, \lg P_{N}\right) \mathrm{d}\left(\lg P_{N}\right)
\end{aligned}
$$

$$
\begin{aligned}
& f\left(\lg p_{1}, \lg p_{2}, \lg P_{N}\right)=f\left(\lg C=\lg P_{N}-\lg p_{2}\right) \\
& \cdot f\left(\lg K=\lg p_{1}+\lg P_{N}-\lg p_{2}\right) f\left(\lg P_{N}\right) \\
& \sum_{i=1}^{n_{1}} w_{i}=1
\end{aligned}
$$

where $f(\cdot)$ is the probability density function.

Secondly, for the $i^{\text {th }}$ cluster of $p_{1}$ and $p_{2}, n_{2}$ clusters are classified according to the probability density function of $P_{N}$, with weight $w_{i j}$ of each cluster, as defined by (57) and (58).

$w_{i j} \propto f\left(\lg P_{N} \mid \lg p_{1}, \lg p_{2}\right) \propto f\left(\lg p_{1}, \lg p_{2}, \lg P_{N}\right)$

$\sum_{j=1}^{n_{2}} w_{i j}=1$

\subsection{Aggregate model of heterogeneous loads}

It can be seen that there are $n_{1} \times n_{2}$ clusters of $C, K$ and $P_{N}$, and only $n_{1}$ homogeneous aggregate models are calculated. The number of air conditioners of the $i^{\text {th }}$ cluster in the on state $X_{i j}(t)$ is subject to the binomial distribution:

$X_{i j}(t) \sim B\left(N_{a c} w_{i} w_{i j}, \bar{m}_{i j}(t)\right)$

The average electrical power consumption of an individual air conditioner is:

$P(t)=\frac{1}{N_{a c}} \sum_{i=1}^{n_{1}} \sum_{j=1}^{n_{2}} P_{N_{j}} X_{i j}(t)$

where $P_{N, j}$ is the rated power of the air conditioner in the $j^{\text {th }}$ cluster.

The expectation and variance of the average electrical power are:

$E(P(t))=\sum_{i=1}^{n_{1}} \sum_{j=1}^{n_{2}} P_{N_{j} j} w_{i} w_{i j} \bar{m}_{i j}(t)$

$D(P(t))=\frac{1}{N_{a c}} \sum_{i=1}^{n_{1}} \sum_{j=1}^{n_{2}} P_{N_{j}}^{2} w_{i} w_{i j} \bar{m}_{i j}(t)\left(1-\bar{m}_{i j}(t)\right)$

Considering that the binomial distribution can be approximated by the normal distribution and the linear
Table 1 Model parameter values

\begin{tabular}{ll}
\hline Parameter & Value \\
\hline Average thermal conductance $K$ & $500 \mathrm{~W} /{ }^{\circ} \mathrm{C}$ \\
Average thermal capacitance $C$ & $3.6 \times 10^{7} \mathrm{~J} /{ }^{\circ} \mathrm{C}$ \\
Average energy transfer rate $P_{h}$ & $1.4 \times 10^{4} \mathrm{~W}$ \\
COP $\alpha$ & 2.5 \\
Temperature set point $x_{\text {set }}$ & $20{ }^{\circ} \mathrm{C}$ \\
Thermostat dead band $\delta$ & $0.5{ }^{\circ} \mathrm{C}$ \\
Temperature outside the space $x_{o}$ & $32{ }^{\circ} \mathrm{C}$ \\
Noise standard deviation $\sigma$ & $0.01{ }^{\circ} \mathrm{C} \cdot \mathrm{s}^{-\frac{1}{2}}$ \\
Standard deviation of lognormal distributions $\sigma_{p}$, & 0.2 \\
as a fraction of mean value, for $K, C$ and $P_{h}$ & \\
\hline
\end{tabular}

combination of normal distributions is also a normal distribution [25], the average electrical power consumption of an individual air conditioner has approximately a normal distribution:

$P(t) \sim N(E(P(t)), D(P(t)))$

The point estimation and the interval estimation of power consumption of heterogeneous loads can be obtained based on this normal distribution.

\section{Simulation results}

The thermal model parameters, which are chosen using [21], are shown in Table 1. Numerical methods in Sections 3 and 4 are conducted separately based on these parameters. The Monte Carlo method is applied to verify the effectiveness of the methods. It is assumed that the temperature set point undergoes a positive $0.2{ }^{\circ} \mathrm{C}$ step change at time $5000 \mathrm{~s}$.

\subsection{Results of FDM}

The time step $\tau=1 \mathrm{~s}$ and the temperature step $h=0.01{ }^{\circ} \mathrm{C}$ are chosen according to (43). The initial states can be acquired by calculating the stationary solutions before the temperature set point is changed using the proposed methods in Section 3.3. The results of the FDM, which are the probability density of air conditioners in the off and on states, are shown in Fig. 1a and Fig. 1b, respectively. It can be observed that the probability density of air conditioners in the off state firstly climbs up, and then declines after $5000 \mathrm{~s}$. The trend of the probability density of air conditioners in the on state is opposite after $5000 \mathrm{~s}$.

Figure 2 shows the proportion of air conditioners in the on state. The proportion of air conditioners in the on state 


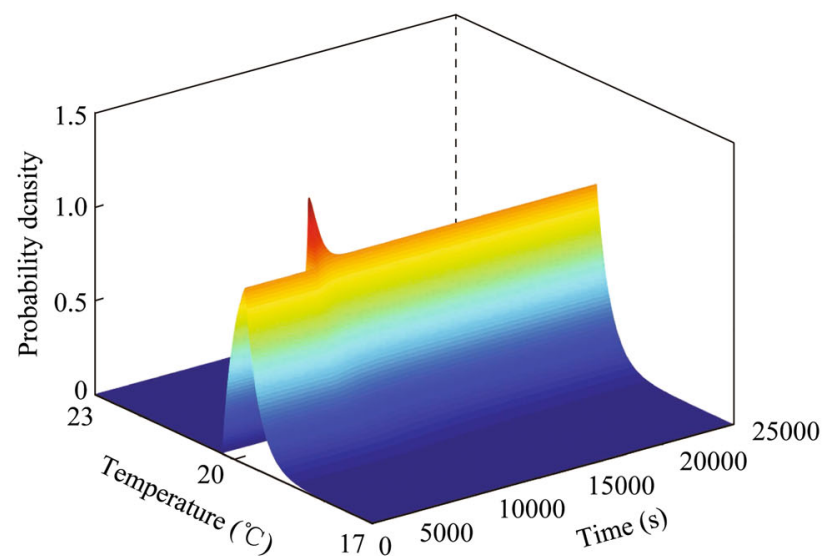

(a) Off state

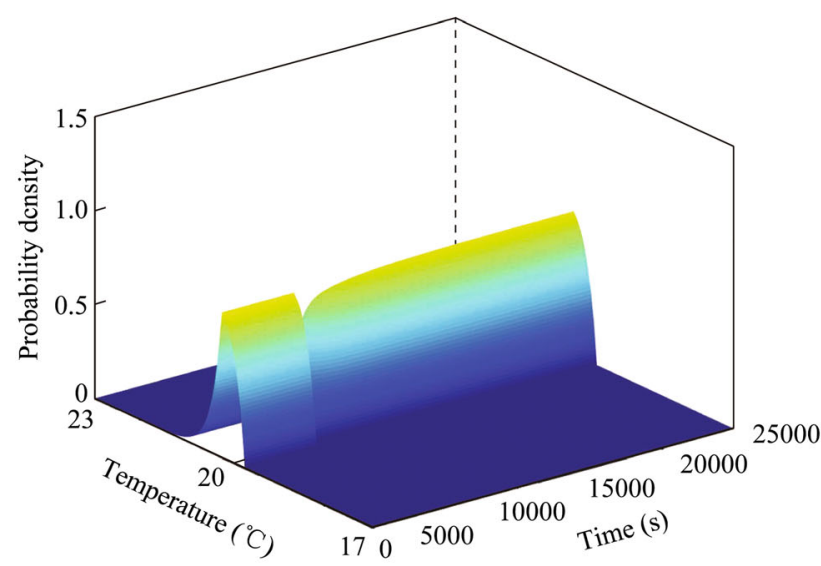

(b) On state

Fig. 1 Probability density of air conditioners in off and on states

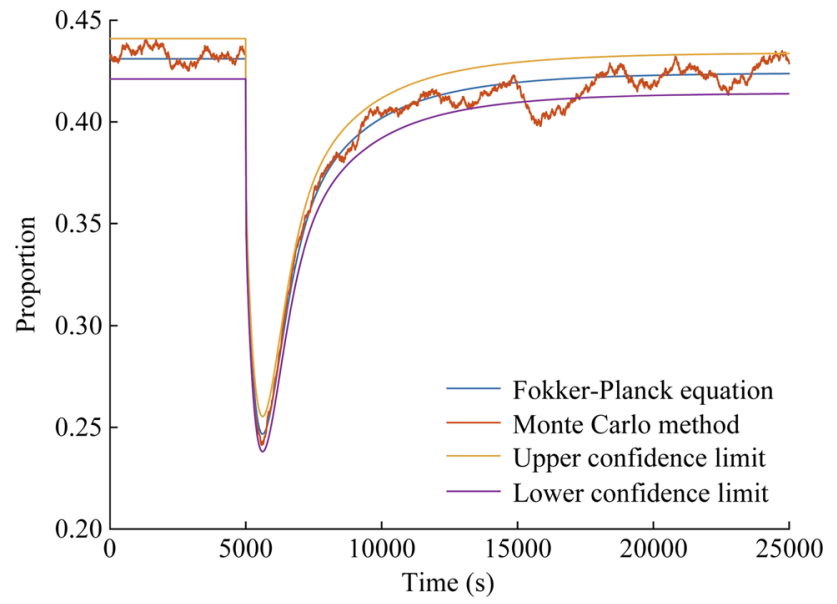

Fig. 2 Proportion of air conditioners in on state with 10000 air conditioners

firstly decreases when the step change is introduced at $5000 \mathrm{~s}$, and then increases afterwards. The stable value that it reaches is smaller than the value before, which matches the expectation. To verify the accuracy of the FDM, the

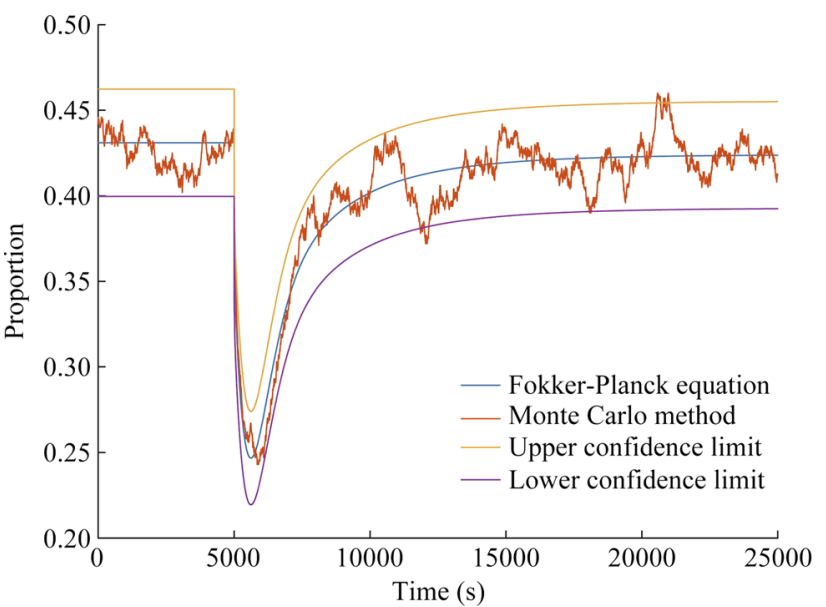

(a) 1000 air conditioners

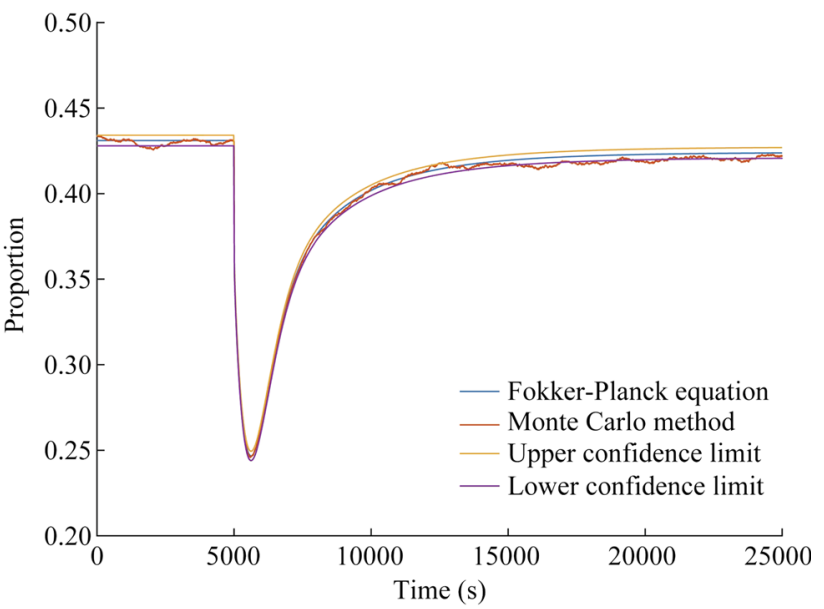

(b) 100000 air conditioners

Fig. 3 Proportion of air conditioners in on state with 1000 and 100000 air conditioners

Monte Carlo method is implemented. From Fig. 2, we can see that the results of Monte Carlo method are around the results of the FDM, which indicates that the results of the FDM are accurate. To obtain the fluctuation behavior of the proportion, the confidence coefficient for interval estimation is set as $95 \%$, and the upper and lower confidence limits are also illustrated in Fig. 2. The majority of results of the Monte Carlo method are within the confidence interval, which verifies the effectiveness of interval estimation.

This paper also examines the different fluctuation behaviors of various amounts of air conditioners. The proportion of air conditioners in the on state is compared for 1000 and 100000 air conditioners in Fig. 3a and Fig. 3b, respectively. It can be seen that point estimations (the mean values) do not change with different amounts of air conditioners. But the Monte Carlo results show that the fluctuation is more obvious when the number of air conditioners is small. The interval estimates are also shown 


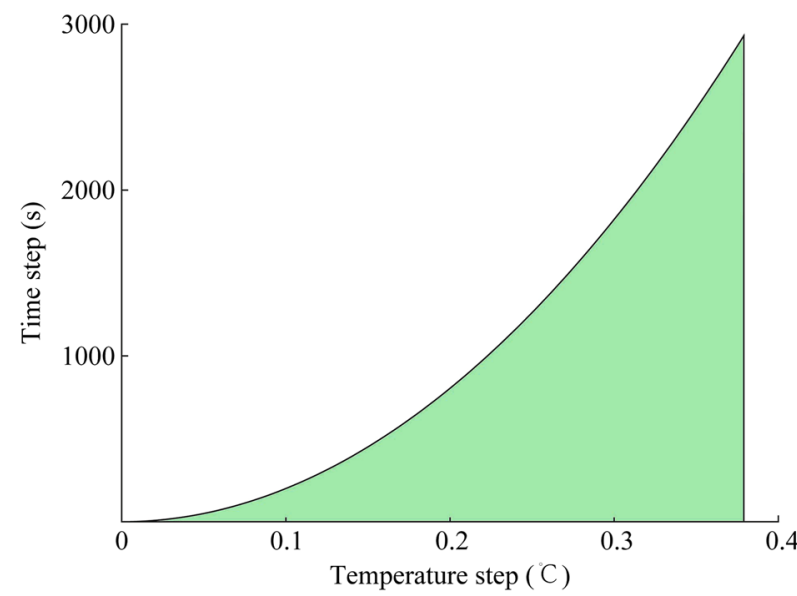

(a) Proper grid spacing zone

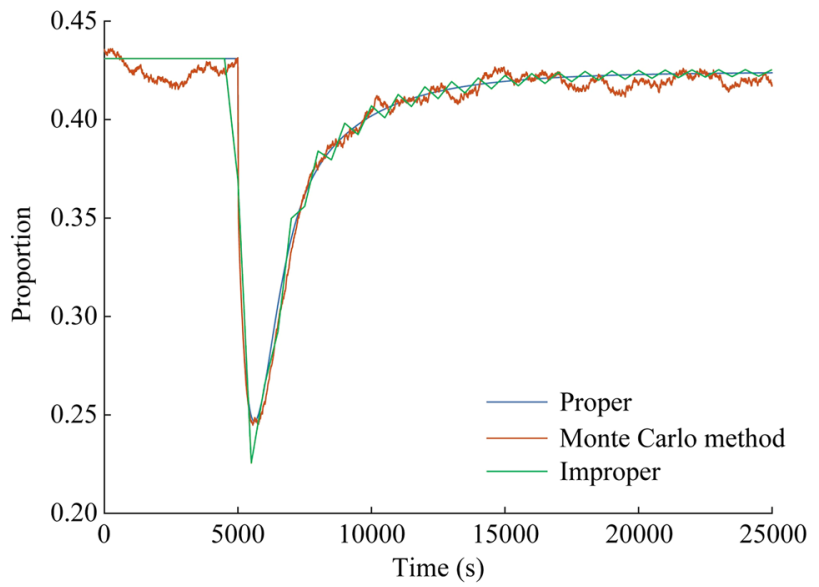

(b) Numerical results with proper and improper grid spacings

Fig. 4 Influence of grid spacings when thermal capacity is large

and they correctly estimate the fluctuation behavior of the Monte Carlo results.

\subsection{Influence of grid spacings}

The choice of grid spacings is important to achieve accurate results from numerical methods. The proper grid spacing zone is shown by the green area of Fig. $4 \mathrm{a}$ according to (43). Because the criterion is a sufficient condition for the stability of the numerical method, the numerical results are not necessarily inaccurate with grid spacings that are outside this zone. For example, the time step $\tau=100 \mathrm{~s}$ with the temperature step $h=0.01{ }^{\circ} \mathrm{C}$ is not in the proper grid spacing zone but the numerical results are still accurate, because the difference scheme remains quite stable. However, if the grid spacings are too large, such as the time step $\tau=500 \mathrm{~s}$ and the temperature step $h=0.01{ }^{\circ} \mathrm{C}$, the numerical results will be inaccurate, as indicated in Fig. 4b.

The choice of grid spacing is particularly important when the thermal capacity is small. For example, consider

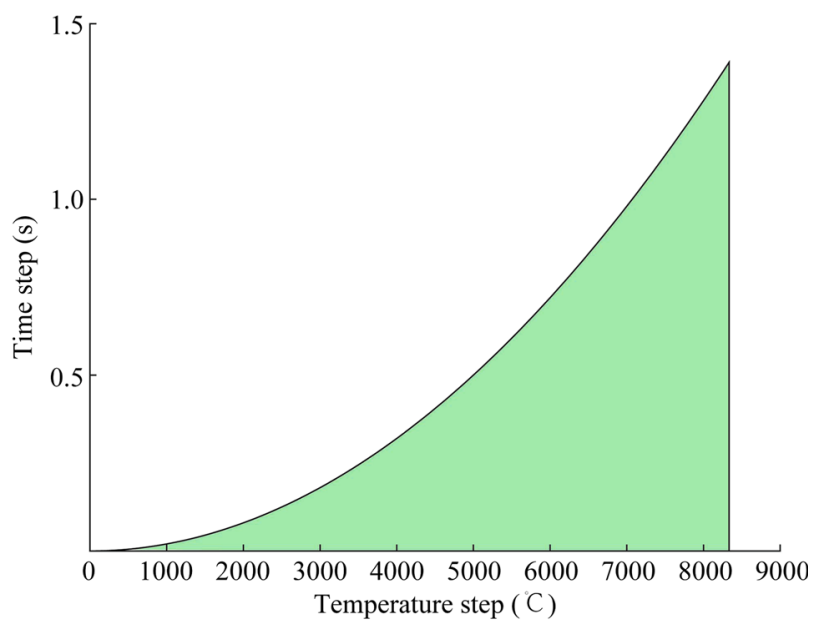

(a) Proper grid spacing zone

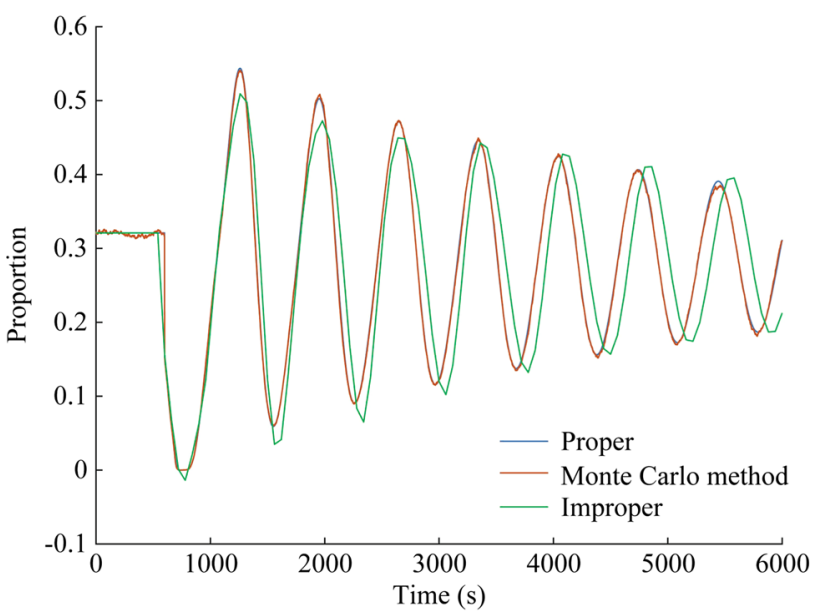

(b) Numerical results with proper and improper grid spacings

Fig. 5 Influence of grid spacings when the thermal capacity is small a simulation in which the thermal capacity is $C=10^{6} \mathrm{~J} /$ ${ }^{\circ} \mathrm{C}$, the set point is $x_{\text {set }}=23{ }^{\circ} \mathrm{C}$, the thermostat dead band is $\delta=2{ }^{\circ} \mathrm{C}$ and the other parameters are the same as in Table 1. It is assumed that the set point undergoes a positive $1{ }^{\circ} \mathrm{C}$ step change at $600 \mathrm{~s}$. The proper grid spacing zone is shown in the filled area of Fig. 5a and the numerical simulation results are shown in Fig. $5 \mathrm{~b}$. It can be seen that, when the time step is $\tau=60 \mathrm{~s}$ and the temperature step is $h=0.01{ }^{\circ} \mathrm{C}$, the results are inaccurate and sometimes less than 0 , which is not physically correct.

\subsection{Stationary solutions}

Before changing the set point, stationary solutions in the off and on states are shown in Fig. 6a and Fig. 6b, respectively. It can be observed that analytical and numerical solutions are almost coincident, which supports the correctness of both methods. The stationary solutions for the scenario of small thermal capacity, developed in Section 5.2, are shown before changing the set point in 


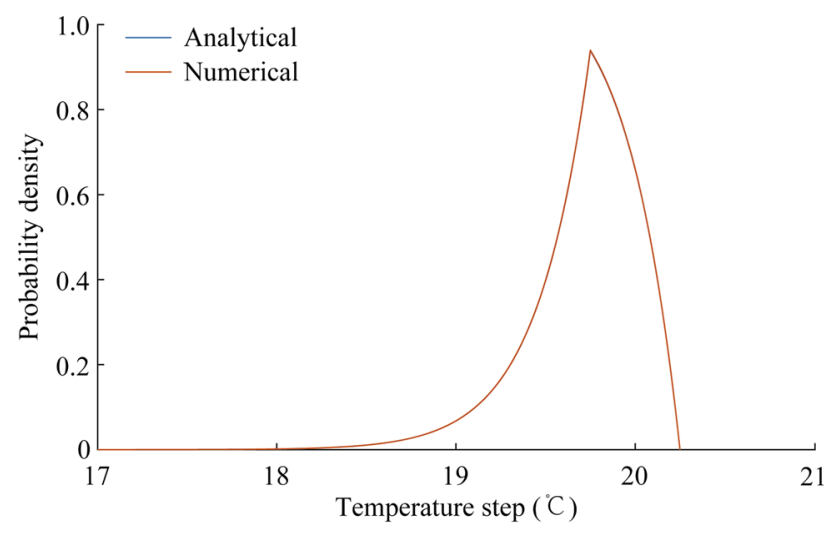

(a) Off state

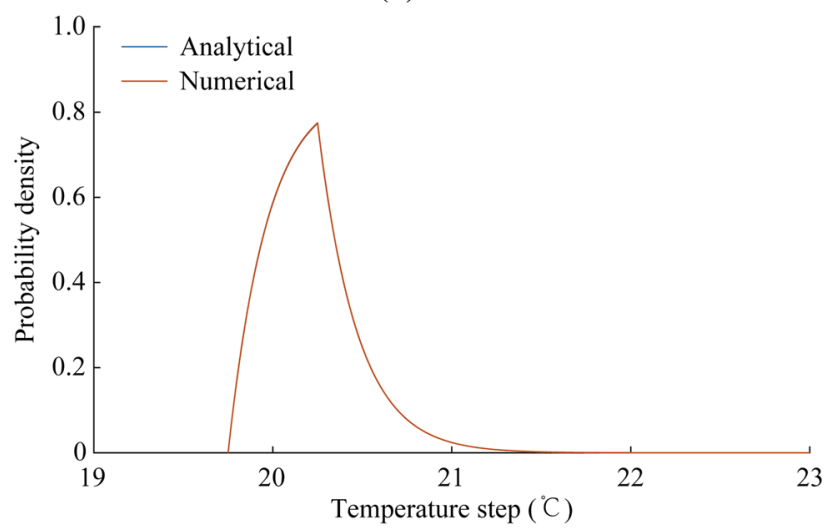

(b) On state

Fig. 6 Stationary solutions in off and on states based on large thermal capacitance before changing set point

Fig. 7a and Fig. 7b. These results also verify the methods proposed in Section 3.3.

\subsection{Results for heterogeneous loads}

In this paper, the load heterogeneity is modelled by varying the parameters $C, K$ and $P_{N}$. These are the fundamental parameters of the space thermal model and they obey lognormal distributions [21]. It should be noted that the parameters are independent, although for the purpose of clustering a dimension reduction is used in Section 4.1. The mean and variance of each parameter have been shown in Table 1. Here we provide a parameter sensitivity analysis based on changes of heterogeneous parameters. The influence of parameters is evaluated by root mean square errors (RMSEs) between the results of Monte Carlo simulation using heterogeneous parameters and the results of the Fokker-Planck equations using average parameters. The sensitivity analysis results are shown in Fig. 8. It can be seen that the parameters $K$ and $P_{N}$ have a greater impact on the results than the parameter $C$. The scale of impact suggests that it is necessary to consider heterogeneous parameters.

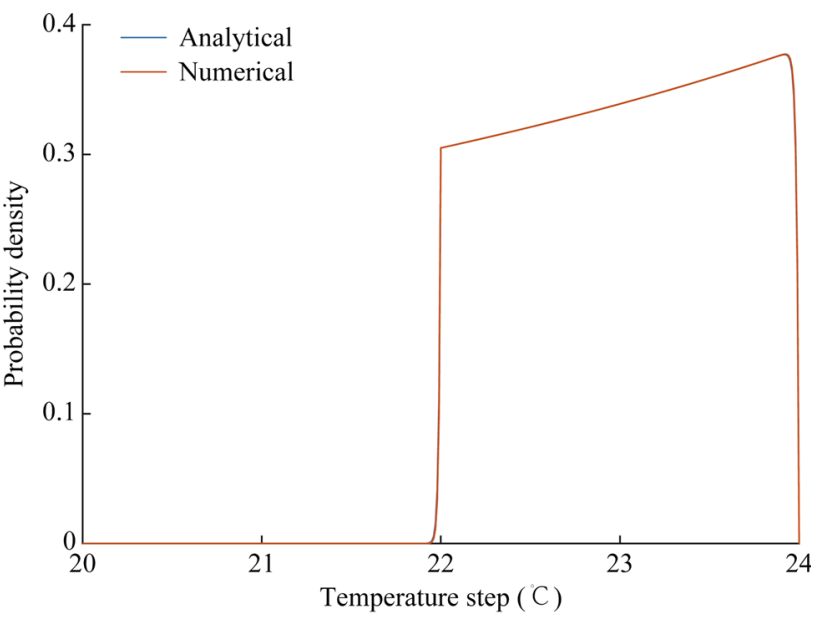

(a) Off state

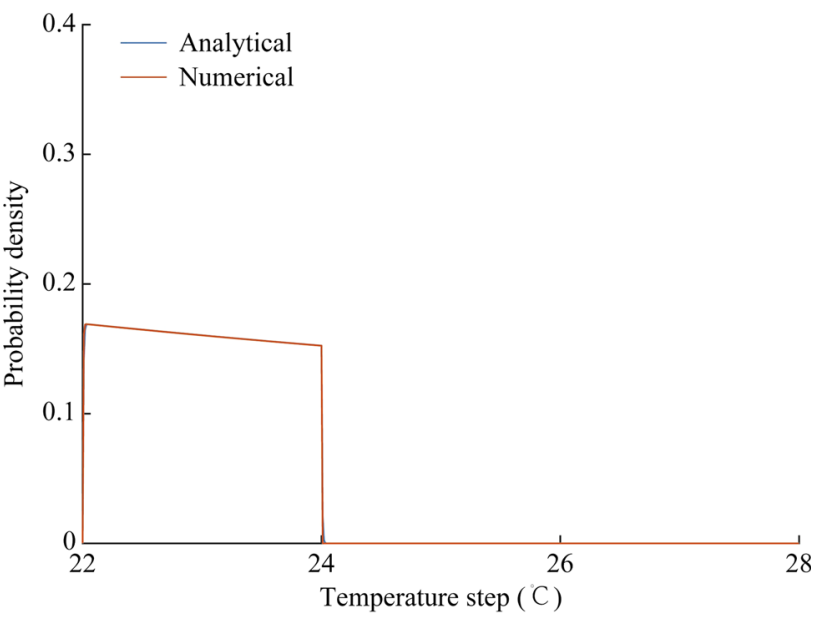

(b) On state

Fig. 7 Stationary solutions in off and on states based on small thermal capacitance before changing set point

Figure 9 shows the average electrical power consumption of an individual air conditioner considering heterogeneous parameters. The results of Monte Carlo method are used as the reference. We can see that the results of the FDM are accurate and reliably indicate the confidence interval. The confidence coefficient of interval estimation is set as $95 \%$ and the upper and lower confidence limits are illustrated in Fig. 9.

\subsection{Application to regulation services}

In Section 3.2, linear state equations are developed by solving CFPEs using FDM, which is helpful for designing control systems. The proposed aggregate model is applied to regulation services. The control strategy proposed in [14] is used in this paper. The aggregator collects information about air conditioning loads, such as their working state ("on" or "off"), the indoor temperature and so on. The control signal is decided by the aggregate model and 


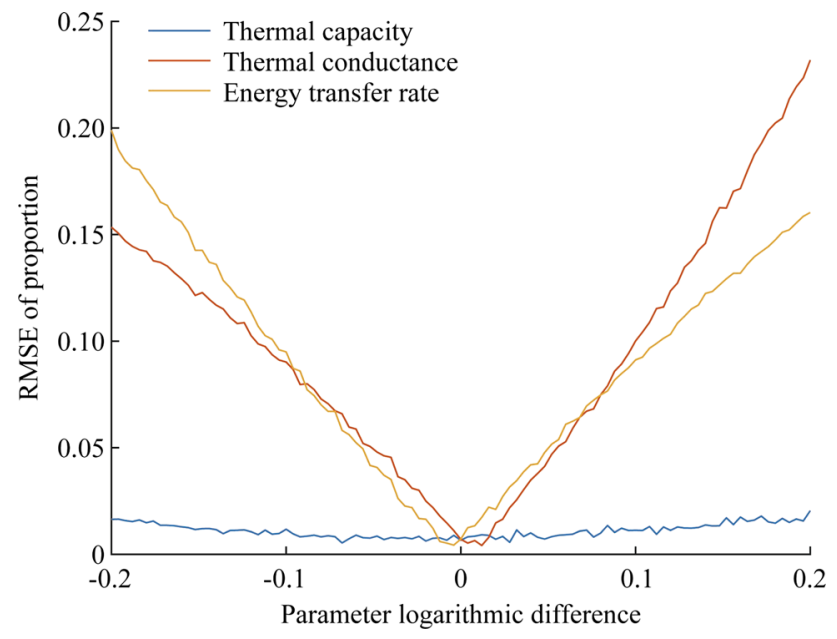

Fig. 8 Parameter sensitivity analysis

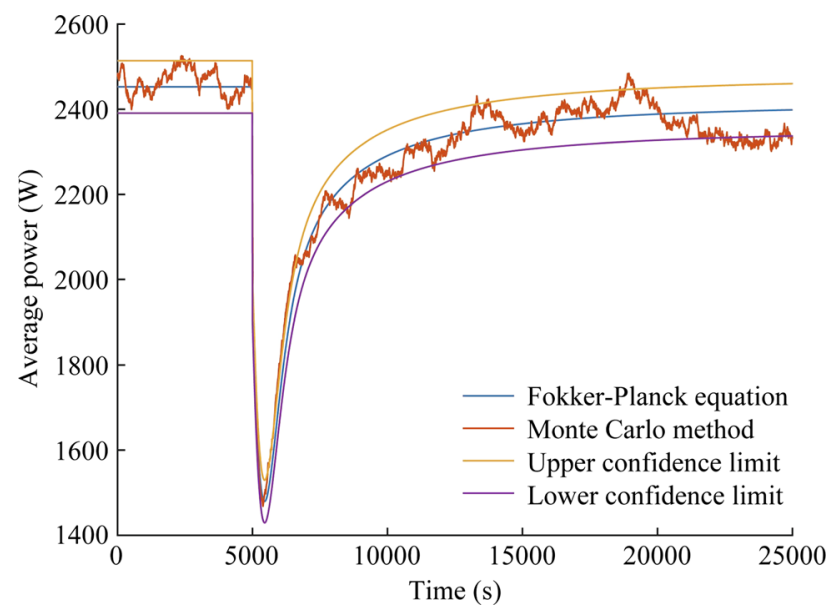

Fig. 9 Average electrical power consumption of an individual air condition among a total population of 10000

the control strategy includes a lock time constraint to avoid short-cycling of devices. The control signal, which is a scalar $\rho \in[-1,1]$, is broadcast to the controlled air conditioners. If an air conditioner is working in the "off" state and $\rho$ is positive, the air conditioner will have probability $\rho$ of turning "on" provided it is not locked. If the air conditioner is working in the "on" state and $\rho$ is negative, the air conditioner will have probability $-\rho$ of turning "off".

The number of controlled air conditioners is 1000 and their total load power capacity is about 5.6 MW. The stationary power of air conditioning loads before control is about 2.4 MW. The lockout time for changing the state of air conditioners is set as 5 minutes and the control interval is 30 seconds. We choose the regulation signal from time 12:00 to $12: 40$ on 1 June 2016 available on the PJM website [28] and the range of the regulation signal is adjusted to $4 \mathrm{MW}$. The Monte Carlo method is used to

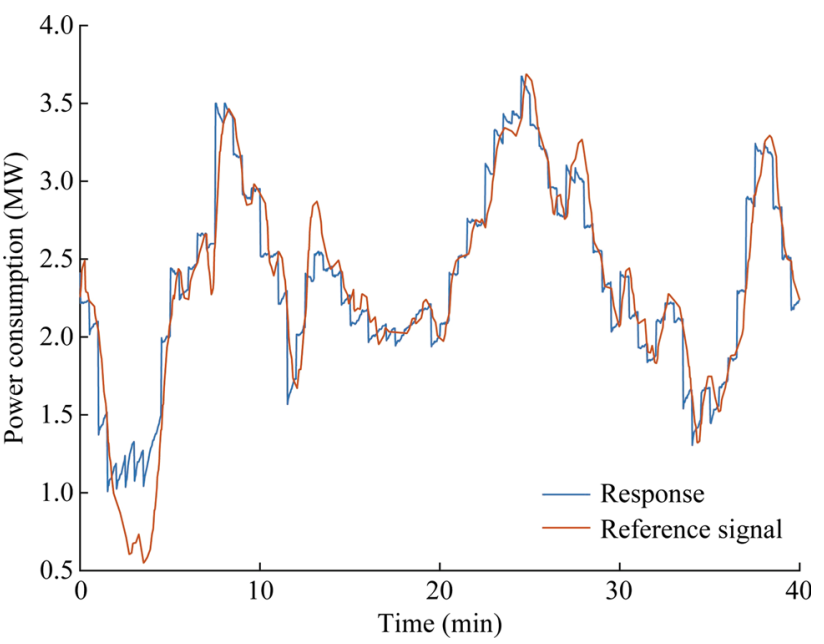

Fig. 10 Tracking performance of following regulation signal

simulate the scenario. The tracking response is illustrated in Fig. 10. It can be seen that the aggregate power of the air conditioning loads can follow the reference signal well. However, the performance is poorer at some time points due to the influence of lock time constraint.

\section{Conclusion}

In this paper, CFPEs are applied to aggregate air conditioning loads. The aggregate model using Fokker-Planck equations is accurate, however this model was not suitable for control since the partial differential equations are difficult to solve. The derivations in this paper successfully apply the aggregate model using Fokker-Planck equations to control design. Compared with other aggregate models, the aggregate model using Fokker-Planck equations takes the randomness of air conditioning loads into consideration, leading to practical solutions.

Because the analytical solutions of CFPEs cannot be derived at present, linear state equations, which characterize the temperature probability density evolution of the air conditioner population considering randomness, are developed by solving CFPEs using the finite difference method (FDM). The grid spacings of difference scheme are obtained by analyzing numerical stability and convergence based on the maximal principle. Both analytical and numerical methods are used to obtain stationary solutions.

Considering that the parameters may be heterogeneous, a classification method using dimension reduction is proposed. To avoid the limitations of point estimation, interval estimation is applied to describe the stochastic behavior according to the normal distribution, which is used to approximate the binomial distribution. Simulation results 
of point estimation and interval estimation are verified by Monte Carlo simulation. The selection of appropriate grid spacings is shown to be important for numerical methods.

Regarding stationary solutions, the simulation results of analytical and numerical methods are almost the same, indicating that the methods proposed in the paper are effective. The aggregate model is successfully applied to an existing control strategy to follow the reference signal for regulation services, and the performance is good. In the future, new control strategies for air conditioning loads can be developed using the proposed methods.

Acknowledgements This work was supported by National Natural Science Foundation of China (No. 51177093).

Open Access This article is distributed under the terms of the Creative Commons Attribution 4.0 International License (http:// creativecommons.org/licenses/by/4.0/), which permits unrestricted use, distribution, and reproduction in any medium, provided you give appropriate credit to the original author(s) and the source, provide a link to the Creative Commons license, and indicate if changes were made.

\section{Appendix A}

$\boldsymbol{A}_{1}$ from Section 3.2 is expressed as follows:

$$
\boldsymbol{A}_{1}=\left[\begin{array}{ll}
\boldsymbol{A}_{11} & \boldsymbol{A}_{12} \\
\boldsymbol{A}_{13} & \boldsymbol{A}_{14}
\end{array}\right]
$$

where $\boldsymbol{A}_{11}$ is a $\left(N_{a}+N_{b}\right) \times\left(N_{a}+N_{b}\right)$ tridiagonal matrix. Since the implicit central finite-difference scheme is applied to solve CFPEs, the value of the following $\theta$ is 0.5 .

$$
\begin{aligned}
& \boldsymbol{A}_{11}=\left[\begin{array}{ccccc}
b_{11}(1) & c_{11}(1) & & & \\
& \ddots & & & \\
& a_{11}(i) & b_{11}(i) & c_{11}(i) & \\
& & & \ddots & \\
& & & a_{11}(n) & b_{11}(n)
\end{array}\right] \\
& a_{11}(i)= \begin{cases}\theta\left(\frac{F_{0}\left(x_{i-1}^{a}\right)}{2 h}-\frac{\sigma^{2}}{2 h^{2}}\right) & 1<i<N_{a}+1 \\
\theta\left(\frac{F_{0}\left(x_{-}\right)}{h}-\frac{\sigma^{2}}{h^{2}}\right) & i=N_{a}+1 \\
\theta\left(\frac{F_{0}\left(x_{i-N_{a}-1}^{b}\right)}{2 h}-\frac{\sigma^{2}}{2 h^{2}}\right) & N_{a}+1<i \leq N_{a}+N_{b}\end{cases}
\end{aligned}
$$

$$
\begin{aligned}
& b_{11}(i)= \begin{cases}\frac{1}{\tau}-\theta\left(\frac{F_{0}\left(x_{\min }\right)}{h}+\frac{K}{C}-\frac{\sigma^{2}}{h^{2}}\right) & i=1 \\
\frac{1}{\tau}-\theta\left(\frac{K}{C}-\frac{\sigma^{2}}{h^{2}}\right) & 1<i \leq N_{a}+N_{b} \text { and } i \neq N_{a}+1 \\
\frac{2}{\tau}-2 \theta\left(\frac{K}{C}-\frac{\sigma^{2}}{h^{2}}\right) & i=N_{a}+1\end{cases} \\
& c_{11}(i)= \begin{cases}-\theta\left(\frac{F_{0}\left(x_{\min }\right)}{h}+\frac{\sigma^{2}}{h^{2}}\right) & i=1 \\
-\theta\left(\frac{F_{0}\left(x_{i-1}^{a}\right)}{2 h}+\frac{\sigma^{2}}{2 h^{2}}\right) & 1 \leq i<N_{a}+1 \\
-\theta\left(\frac{F_{0}\left(x_{-}\right)}{h}+\frac{\sigma^{2}}{h^{2}}\right) & i=N_{a}+1 \\
-\theta\left(\frac{F_{0}\left(x_{i-N_{a}-1}^{b}\right)}{2 h}+\frac{\sigma^{2}}{2 h^{2}}\right) & N_{a}+1<i<N_{a}+N_{b}\end{cases}
\end{aligned}
$$

$\boldsymbol{A}_{12}$ is a $\left(N_{a}+N_{b}\right) \times\left(N_{b}+N_{c}\right)$ matrix and the only nonzero element is:

$\boldsymbol{A}_{12}\left(N_{a}+1,1\right)=-\theta\left(\frac{F_{1}\left(x_{-}\right)}{h}+\frac{\sigma^{2}}{h^{2}}\right)$

$\boldsymbol{A}_{13}$ is a $\left(N_{b}+N_{c}\right) \times\left(N_{a}+N_{b}\right)$ matrix and the only nonzero element is:

$\boldsymbol{A}_{13}\left(N_{b}, N_{a}+N_{b}\right)=\theta\left(\frac{F_{0}\left(x_{+}\right)}{h}-\frac{\sigma^{2}}{h^{2}}\right)$

$\boldsymbol{A}_{14}$ is a $\left(N_{b}+N_{c}\right) \times\left(N_{b}+N_{c}\right)$ tridiagonal matrix:

$\boldsymbol{A}_{14}=\left[\begin{array}{ccccc}b_{14}(1) & c_{14}(1) & & & \\ & \ddots & & & \\ & a_{14}(i) & b_{14}(i) & c_{14}(i) & \\ & & & \ddots & \\ & & & a_{14}(n) & b_{14}(n)\end{array}\right]$

$$
a_{14}(i)= \begin{cases}\theta\left(\frac{F_{1}\left(x_{i}^{b}\right)}{2 h}-\frac{\sigma^{2}}{2 h^{2}}\right) & 1<i<N_{b} \\ \theta\left(\frac{F_{1}\left(x_{+}\right)}{h}-\frac{\sigma^{2}}{h^{2}}\right) & i=N_{b} \\ \theta\left(\frac{F_{1}\left(x_{i-N_{b}}^{c}\right)}{2 h}-\frac{\sigma^{2}}{2 h^{2}}\right) & N_{b}<i<N_{b}+N_{c} \\ \theta\left(\frac{F_{1}\left(x_{\max }\right)}{h}-\frac{\sigma^{2}}{h^{2}}\right) & i=N_{b}+N_{c}\end{cases}
$$




$$
b_{14}(i)= \begin{cases}\frac{2}{\tau}-2 \theta\left(\frac{K}{C}-\frac{\sigma^{2}}{h^{2}}\right) & i=N_{b} \\ \frac{1}{\tau}-\theta\left(\frac{K}{C}-\frac{\sigma^{2}}{h^{2}}\right) & 1 \leq i<N_{b}+N_{c} \text { and } i \neq N_{b} \\ \frac{1}{\tau}-\theta\left(-\frac{F_{1}\left(x_{\max }\right)}{h}+\frac{K}{C}-\frac{\sigma^{2}}{h^{2}}\right) & i=N_{b}+N_{c}\end{cases}
$$

$$
c_{14}(i)= \begin{cases}-\theta\left(\frac{F_{1}\left(x_{i}^{b}\right)}{2 h}+\frac{\sigma^{2}}{2 h^{2}}\right) & 1 \leq i<N_{b} \\ -\theta\left(\frac{F_{1}\left(x_{+}\right)}{h}+\frac{\sigma^{2}}{h^{2}}\right) & i=N_{b} \\ -\theta\left(\frac{F_{1}\left(x_{i-N_{b}}^{c}\right)}{2 h}+\frac{\sigma^{2}}{2 h^{2}}\right) & N_{b}+1<i<N_{b}+N_{c}\end{cases}
$$

$\boldsymbol{A}_{2}$ is almost the same as $\boldsymbol{A}_{1}$ when $\theta$ is changed to $\theta-1$.

\section{References}

[1] Wood AJ, Wollenberg BF (1996) Power generation, operation, and control, 2nd edn. Wiley, New York

[2] Xu Z, Ostergaard J, Togeby M (2011) Demand as frequency controlled reserve. IEEE Trans Power Syst 26(3):1062-1071

[3] Wang K, Chu X, Zhang W (2015) Cooperative optimization for supply/demand side reserve by decoupling cost and value of reserve. Proc CSEE 35(20):5188-5198

[4] Callaway DS, Hiskens IA (2011) Achieving controllability of electric loads. Proc IEEE 99(1):184-199

[5] Al-Rabghi OM, Akyurt MM (2004) A survey of energy efficient strategies for effective air conditioning. Energy Convers Manag 45(11):1643-1654

[6] Song M, Gao C, Su W (2016) Modeling and controlling of airconditioning load for demand response applications. Autom Electr Power Syst 40(14):158-167

[7] Liu M, Shi Y, Liu X (2016) Distributed MPC of aggregated heterogeneous thermostatically controlled loads in smart grid. IEEE Trans Ind Electron 63(2):1120-1129

[8] Hao H, Sanandaji BM, Poolla K et al (2015) Aggregate flexibility of thermostatically controlled loads. IEEE Trans Power Syst 30(1):189-198

[9] Liu M, Chu X, Zhang W et al (2014) Dispatch and control strategies for air-conditioning load groups based on maintenance of load diversity. Proc CSEE 34(22):3674-3682

[10] Molina-Garcia A, Kessler M, Fuentes JA et al (2011) Probabilistic characterization of thermostatically controlled loads to model the impact of demand response programs. IEEE Trans Power Syst 26(1):241-251

[11] Koch S, Mathieu JL, Callaway DS (2011) Modeling and control of aggregated heterogeneous thermostatically controlled loads for ancillary services. In: Proceedings of the 2011 power systems computation conference, Stockholm, Sweden, 22-26 August 2011, pp 1-7

[12] Mathieu JL, Koch S, Callaway DS (2013) State estimation and control of electric loads to manage real-time energy imbalance. IEEE Trans Power Syst 28(1):430-440
[13] Zhao H, Wu Q, Huang S et al (2016) Hierarchical control of thermostatically controlled loads for primary frequency support. IEEE Trans Smart Grid. https://doi.org/10.1109/tsg.2016. 2624509

[14] Zhang W, Lian J, Chang CY et al (2013) Aggregated modeling and control of air conditioning loads for demand response. IEEE Trans Power Syst 28(4):4655-4664

[15] Vrettos E, Ziras C, Andersson G (2017) Fast and reliable primary frequency reserves from refrigerators with decentralized stochastic control. IEEE Trans Power Syst 32(4):2924-2941

[16] Xu Z, Callaway DS, Hu Z et al (2016) Hierarchical coordination of heterogeneous flexible loads. IEEE Trans Power Syst 31(6):4206-4216

[17] Perfumo C, Kofman E, Braslavsky JH et al (2012) Load management: model-based control of aggregate power for populations of thermostatically controlled loads. Energy Convers Manag 55:36-48

[18] Wei W, Wang D, Jia $\mathrm{H}$ et al (2017) Hierarchical and distributed demand response control strategy for thermostatically controlled appliances in smart grid. J Mod Power Syst Clean Energy 5(1):30-42

[19] Malhamé R, Chong CY (1985) Electric load model synthesis by diffusion approximation of a high-order hybrid-state stochastic system. IEEE Trans Autom Control 30(9):854-860

[20] Malhamé R, Chong CY (1988) On the statistical properties of a cyclic diffusion process arising in the modeling of thermostatcontrolled electric power system loads. SIAM J Appl Math 48(2):465-480

[21] Callaway DS (2009) Tapping the energy storage potential in electric loads to deliver load following and regulation, with application to wind energy. Energy Convers Manag 50(5):1389-1400

[22] Ralston A, Wilf HS (1960) Mathematical methods for digital computers. Wiley, New York

[23] Mortensen RE, Haggerty KP (1988) A stochastic computer model for heating and cooling loads. IEEE Trans Power Syst 3(3): 1213-1219

[24] Alvarez C, Malhamé R, Gabaldon A (1992) A class of models for load management application and evaluation revisited. IEEE Trans Power Syst 7(4):1435-1443

[25] Devore J (2008) Probability and statistics for engineering and the sciences, 7 th edn. Cengage Learning, Toronto

[26] Morton KW, Mayers DF (2005) Numerical solution of partial differential equations: an introduction, 2nd edn. Cambridge University Press, Cambridge

[27] Cheng D, Zhang W, Liu J (2017) Window-varying particle filter for parameter identification of space thermal model. IEEE Trans Instrum Meas 66(1):165-176

[28] PJM Markets \& Operations (2017) Market-based regulation in ancillary service. http://www.pjm.com/ /media/markets-ops/ ancillary/mkt-based-regulation/regulation-data.asha. Accessed 22 January 2017

Dingyi CHENG received the B.S. degree in Electrical Engineering from Shandong University, China, in 2013. He is currently pursuing the Ph.D. degree in the School of Electrical Engineering, Shandong University. He is also with the Key Laboratory of Power System Intelligent Dispatch and Control of Ministry of Education, Shandong University. His research interests are power system analysis and optimization, and demand-side response. 
Wen ZHANG received the B.S., M.S. and Ph.D. degrees all in Electrical Engineering from Shandong University, China, in 1989, 1992 and 2006, respectively. She is currently a professor in the School of Electrical Engineering, Shandong University. Her research interests are power system analysis and control, demand-side response and application of intelligent methods to power systems.
Yutian LIU received the Ph.D. degree from Xi'an Jiaotong University, China, in 1994. He is the Taishan chair professor of School of Electrical Engineering, Shandong University, China. His research interests include power system stability and restoration, smart grid and renewable energy sources integration. 